\title{
Parametric Interactions of Optical Modes
}

\author{
AMNON YARIV, MEMBER,IEEE
}

\begin{abstract}
A formalism for treating interactions between optical modes in the presence of time-varying parameters is developed. The problems of parametric oscillation, frequency conversion, and internal laser modulation are treated, as well as a new class of interactions involving parametric modulation in the presence of negative losses.
\end{abstract}

\section{INTRODUCTION}

$\mathrm{T}$ VHIS PAPER is concerned with the study of parametric interactions in the optical region. The concept of parametric interactions is taken to mean the propagation, or oscillation, of electromagnetic waves in the presence of time-varying parameters [1], [2]. These parameters include not only reactive ones, but lossy ones, such as conductivity, as well.

The formalism developed below is relevant to a number of experimental situations that have been the subject of numerous recent investigations. Among these are the the AM phase-locked laser of Hargrove et al. [3]; the FM laser proposed by Yariv [4], [10] and demonstrated by Peterson and Yariv [5] and by Harris and Targ [6]; and the optical parametric oscillator discussed by Kingston [7], Kroll [8], and demonstrated by Giordmaine and Miller [9].

Some of the results derived below have been used, without derivation, by the author in an earlier publication [10].

\section{Expansion of Resonator Fields}

Since a great deal of the analysis that follows is concerned with parametric interactions inside optical resonators (or, in general, any resonator with typical dimensions large compared to the wavelength), it is worthwhile to derive first the spectrum of modes and their characteristic frequencies for the case of a passive resonator. These modes, considered as a complete orthonormal set, will be used to expand the resonator field in the presence of parametric modulation.

A formalism developed by Slater [11] is found convenient for obtaining the mode spectrum discussed above. It is especially useful, since it is not necessary to specify the exact shape of the resonator so that the results obtained are very general.

Using Slater's formalism we define two infinite sets of vector functions $\bar{H}_{a}(\bar{r})$ and $\bar{E}_{a}(\bar{r})$ satisfying

$$
\begin{aligned}
k_{a} \bar{H}_{a}= & \bar{\nabla} \times \bar{H}_{a}, \quad k_{a} \bar{H}_{a}=\bar{\nabla} \times \bar{E}_{a} \\
& \bar{\nabla} \cdot \bar{E}_{a}=\bar{\nabla} \cdot \bar{H}_{a}=0
\end{aligned}
$$

Manuscript received August 17, 1965; revised November 29,1965, The work reported in this paper was supported by the U. S. Air Force Systems Engineering Group under Contract AF33(615)-2800, "Research in Nonlinear Optics".

The author is with the Division of Engineering and Applied Science, California Institute of Technology, Pasadena, Calif. and $\bar{n} \times \bar{E}_{a}=0$ at the boundaries of the resonator. For the moment, $k_{a}$ is considered as a constant, but will be shown to be equal to $\omega_{a} \sqrt{\mu \epsilon}$ where $\omega_{a}$ is $2 \pi$ times the characteristic frequency of the ath mode. It follows from (1) that

$$
\begin{aligned}
& \bar{\nabla}^{2} \bar{H}_{a}+k_{a}^{2} \bar{H}_{a}=0 \\
& \bar{\nabla}^{2} \bar{E}_{a}+k_{a}^{2} \bar{E}_{a}=0 .
\end{aligned}
$$

It also follows from (1) and the boundary condition $\bar{n} \times \bar{E}_{a}=0$ that

$$
\int_{\boldsymbol{v}_{c}} \bar{E}_{a} \cdot \bar{E}_{b} d v=\int_{V_{0}} \bar{H}_{a} \cdot \bar{H}_{b} d v=0 \quad a \not \neq b
$$

where the integration extends over the whole volume of the optical resonator. The derivation of (4) is given by Slater [11]. The mode amplitudes are normalized so that

$$
\int_{V_{\mathrm{c}}} \bar{H}_{a} \cdot \bar{H}_{b} d v=\int_{V_{a}} \bar{E}_{a} \cdot \bar{E}_{b} d v=\delta_{a \grave{b}} .
$$

Using $\bar{E}_{a}$ and $\bar{H}_{a}$ as complete orthonormal sets in which to expand the electromagnetic fields $\bar{E}(\vec{r}, t)$ and $\bar{H}(\bar{r}, t)$ inside the resonator, we can put

$$
\begin{aligned}
& \bar{E}(\bar{r}, t)=-\sum_{a} \frac{1}{\sqrt{\epsilon}} p_{a}(t) \vec{E}_{a}(\bar{r}) \\
& \bar{H}(\bar{r}, t)=\sum_{a} \frac{1}{\sqrt{\mu}} \omega_{a} q_{a}(t) \bar{H}_{a}(\bar{r})
\end{aligned}
$$

where $\mu$ and $\epsilon$ have their customary definitions. For the moment, $\omega_{a}$ is a scalar constant and $p_{a}(t), q_{a}(t)$ represent the time-varying part of the mode fields.

The field hamiltonian, i.e., the total energy, is given by (using mks units)

$$
\Re=\frac{1}{2} \int_{V_{o}}(\epsilon \bar{E} \cdot \bar{E}+\mu \bar{H} \cdot \bar{H}) d v .
$$

Substituting (6) and using (5) leads to a "harmonicoscillator" form of the hamiltonian

$$
\mathfrak{C}=\frac{1}{2} \sum_{a}\left(p_{a}^{2}+\omega_{a}^{2} q_{a}^{2}\right) .
$$

In order to make some more definite statements about $k_{a}, \omega_{a}$, and the interdependence of $p_{a}(t)$ and $q_{a}(t)$, it is necessary to substitute (6a) and (6b) into Maxwell's equations

$$
\begin{gathered}
\bar{\nabla} \times \bar{E}=-\frac{\partial \bar{B}}{\partial t}=-\frac{\partial}{\partial t}(\mu \bar{H}) \\
\bar{\nabla} \times \vec{H}=\frac{\partial \bar{D}}{\partial t}=\frac{\partial}{\partial t}(\epsilon \bar{E}) .
\end{gathered}
$$


The result is

$$
p_{a}(t)=\frac{d}{d t}\left[q_{a}(t)\right]
$$

and

$$
k_{a}=\omega_{a} \sqrt{\mu \epsilon}
$$

and similarly, from (10)

$$
\omega_{a}^{2} q_{a}(t)=-\frac{d}{d t}\left[p_{a}(t)\right]
$$

Solving (11) and (12) simultaneously yields

$$
\begin{aligned}
& q_{a}(t)=\operatorname{Re}\left[q_{a}(0) e^{j \omega_{a} t}\right] \\
& p_{a}(t)=-\operatorname{Im}\left[\omega_{a} q_{a}(0) e^{j \omega_{a} t}\right] .
\end{aligned}
$$

This identifies $\omega_{a}$ and $k_{a}$ as the characteristic (radian) frequency and wave number, respectively, of the athresonator mode.

\section{The Normal Modes}

It is possible to carry out the analysis completely in terms of $p_{a}$ and $q_{a}$, but it is far more convenient, as will become clear in the next section, to introduce a new set. of field coordinates $c_{a}$ and its complex conjugate $c_{a}^{*}$ which are defined by

$$
\begin{aligned}
& c_{a}^{*}(t)=\left(2 \omega_{a}\right)^{-1 / 2}\left[\omega_{a} q_{a}(t)-j p_{a}(t)\right] \\
& c_{a}(t)=\left(2 \omega_{a}\right)^{-1 / 2}\left[\omega_{a} q_{a}(t)+j p_{a}(t)\right]
\end{aligned}
$$

so that

$$
\begin{aligned}
& q_{a}=\left(2 \omega_{a}\right)^{-1 / 2}\left(c_{a}^{*}+c_{a}\right) \\
& p_{a}=j\left(\frac{\omega_{a}}{2}\right)^{1 / 2}\left(c_{a}^{*}-c_{a}\right)
\end{aligned}
$$

Expressing the total energy (8) in terms of $c_{a}^{*}$ and $c_{a}$ gives

$$
\mathfrak{H}=\sum_{a} \omega_{a} c_{a} c_{a}^{*}
$$

The quantity $c_{a} c_{a}^{*} / \hbar$ is equal to the total number of photons in the ath mode. This definition of normal mode amplitudes is a natural one in the study of parametric interactions, since the basic parametric mechanism can be viewed as a "collision" process. in which an integral number of photons at certain frequencies are "annihilated" while a new set of photons of different energies is "created." Expressing the interaction in terms of field coordinates, such as the $c_{a}$ 's , which are related directly to the number of photons, introduces a desired measure of symmetry into the differential equations describing this process and facilitates their solution.

The equations of motion for $c_{a}^{*}$ and $c_{a}$ in a passive cavity are derived by substituting (15) into (11) and (12).

In a quantum mechanical formulation of the problem (see Louisell [1] $\hbar^{-\frac{1}{2}} c_{a}^{*}$ and $\hbar^{-\frac{1}{6}} c_{a}$ correspond to the creation and annihilation boson operators, respectively.
This results in

$$
\begin{aligned}
& \frac{d c_{a}^{*}}{d t}=+j \omega_{a} c_{a}^{*} \\
& \frac{d c_{a}}{d t}=-j \omega_{a} c_{a}
\end{aligned}
$$

so that

$$
\begin{aligned}
& c_{a}(t)=c_{a}(0) e^{-j \omega_{a} t} \\
& c_{a}^{*}(t)=c_{a}^{*}(0) e^{+j \omega_{a} t} .
\end{aligned}
$$

The total energy at time $t$ is given by $\mathfrak{H}=\sum_{a} \omega_{a} c_{a}^{*}(t) c_{b}(t)=$ $\sum_{a} \omega_{a} c_{a}^{*}(\mathbf{0}) c_{a}(0)$ and is thus a constant of the motion.

\section{Dielectric Modulation in a Resonator}

In this section we consider the case of a multimode resonator whose dielectric constant $\epsilon$ is modulated harmonically in time. The spatial variation of $\epsilon$ is left arbitrary. The solution of Maxwell equations for this case can be expressed as a sum of characteristic solutions of the passive (nonmodulated) resonator with time-varying coefficients. These coefficients, taken at a given instant, describe the distribution of the total energy among the various modes.

Maxwell equations are written, in this case, as

$$
\begin{aligned}
& \nabla \times \bar{E}=-\mu \frac{\partial \vec{H}}{\partial t} \\
& \nabla \times \bar{H}=\bar{\imath}+\frac{\partial}{\partial t}[\epsilon(\bar{r}, t) \bar{E}] .
\end{aligned}
$$

Substituting (6) for $\bar{E}$ and $\bar{H}$ in the first equation of (20) gives

$$
p_{a}=\frac{d q_{a}}{d t}
$$

The same substitution in the second equation of (20) results in

$$
\sum_{a} \frac{1}{\sqrt{\mu}} \omega_{a} q_{a} k_{a} \bar{E}_{a}=-\sum_{a} \frac{1}{\sqrt{\epsilon}} \frac{\partial}{\partial t}\left[\epsilon(\bar{r}, t) p_{a}\right] \bar{E}_{a}
$$

where we assume that no conduction current exists, i.e., $\bar{\imath}=0$. The dielectric constant is taken as the sum of a constant term and a modulated term

$$
\epsilon(\bar{r}, t)=\epsilon+\epsilon_{1}(\bar{r}, t) \text {. }
$$

Using (22) in (21) gives

$$
\sum_{a}\left(\frac{1}{\sqrt{\mu}} \omega_{a} k_{a} q_{a}+\sqrt{\epsilon} \frac{d p_{a}}{d t}\right) \bar{E}_{a}=-\sum_{a} \frac{1}{\sqrt{\epsilon}} \frac{\partial}{\partial t}\left(\epsilon_{1} p_{a}\right) \bar{E}_{a}
$$

Taking the scalar product of the last equation with $\vec{E}_{b}$ and integrating over the cavity volume gives

$$
\omega_{a}^{2} q_{a}(t)=-\frac{d p_{a}(t)}{d t}-\sum_{b} \frac{d}{d t}\left(S_{a b} p_{b}\right)
$$

where $S_{a b}$ is defined by

$$
S_{a b}=\int_{V_{0}} \frac{\epsilon_{1}(\bar{r}, t)}{\epsilon} \bar{E}_{a} \cdot \bar{E}_{b} d v .
$$


The equations of motion for the normal-mode amplitudes are obtained by substituting (15) into (21) and (24). The result being

$\frac{d}{d t}\left(c_{a}^{*}+c_{a}\right)=j \omega_{a}\left(c_{a}^{*}-c_{a}\right)$

$\frac{d}{d t}\left(c_{a}^{*}-c_{a}\right)=j \omega_{a}\left(c_{a}^{*}+c_{a}\right)-\sum_{b} \frac{d}{d t}\left[S_{a b} \sqrt{\frac{\omega_{b}}{\omega_{a}}}\left(c_{b}^{*}-c_{b}\right)\right]$

from which we get:

$$
\begin{aligned}
& \frac{d c_{a}^{*}}{d t}=j \omega_{a} c_{a}^{*}-\frac{1}{2} \sum_{b} \frac{d}{d t}\left[S_{a b} \sqrt{\frac{\omega_{b}}{\omega_{a}}}\left(c_{b}^{*}-c_{b}\right)\right] \\
& \frac{d c_{a}}{d t}=-j \omega_{a} c_{a}+\frac{1}{2} \sum_{b} \frac{d}{d t}\left[S_{a b} \sqrt{\frac{\omega_{b}}{\omega_{a}}}\left(c_{b}^{*}-c_{b}\right)\right] .
\end{aligned}
$$

If the dielectric perturbation is time-harmonic it can be written as

$$
\epsilon_{1}(\bar{r}, t)=\epsilon_{1}(\bar{r}) \cos (\omega t+\phi)
$$

and using (25)

$$
S_{a b}=\frac{1}{2} S_{a b}^{0}\left[e^{i(\omega t+\phi)}+e^{-i(\omega t+\phi)}\right]
$$

where

$$
S_{a b}^{0}=\int_{V_{c}} \frac{\epsilon_{1}(\bar{r})}{\epsilon} \bar{E}_{a} \cdot \bar{E}_{b} d v .
$$

Equations (26) show that at the absence of dielectric modulation, $S_{a b}=0$, the mode amplitudes vary as $c_{a}^{*}=c_{a}^{*}(\mathbf{0}) \exp \left(j \omega_{a} t\right)$. This suggests the substitution

$$
\begin{aligned}
c_{a}^{*}(t) & =D_{a}^{*}(t) e^{j \omega_{a} t} \\
c_{a}(t) & =D_{a}(t) e^{-i \omega_{a} t} .
\end{aligned}
$$

Using (28) and (29) in (26) gives

$$
\begin{aligned}
& \frac{d D_{a}^{*}}{d t} e^{j \omega_{a} t}=-\frac{1}{2} \sum_{b} \frac{S_{a b}^{0}}{2} \sqrt{\frac{\omega_{b}}{\omega_{a}}}\left\{\left[e^{i(\omega t+\phi)}+e^{-j(\omega t+\phi)}\right]\right. \\
& \cdot\left(D_{b}^{*} j \omega_{b} e^{j \omega_{b} t}+\frac{d D_{b}^{*}}{d t} e^{j \omega_{b} t}+D_{b} j \omega_{b} e^{-j \omega b t}-\frac{d D_{b}}{d t} e^{-j \omega_{b} t}\right) \\
& \left.+\left(j \omega e^{j(\omega t+\phi)}-j \omega e^{-j(\omega t+\phi)}\right)\left(D_{b}^{*} e^{j \omega_{b} t}-D_{b} e^{-j \omega_{b} t}\right)\right\} \cdot
\end{aligned}
$$

In the following analysis we will make the adiabatic approximation $\dot{D}_{b} \ll j \omega_{b} D_{b}$ and neglect the terms involving $\dot{D}_{b}$ and $\dot{D}_{b}^{*}$ on the right side of $(30)$. We are now in a position to derive the equations pertaining to specific situations.

\section{FM Laser Oscillation}

Assume an optical resonator of length $L$ corresponding to a laser with characteristic_resonance frequencies

$$
\omega_{a}=a \frac{\pi c}{L}
$$

where $a$ is the mode integer that is equal to $2 L / \lambda$. The modulation radian frequency $\omega$ of the dielectric constant is taken as equal to the intermode spacing $\omega_{a+1}-\omega_{a}=$ $\pi c / L$ i.e.,

$$
\omega=\omega_{a+1}-\omega_{a} .
$$

Using this condition in (30) and keeping on the right side only those terms that are multiplied by $e^{j \omega_{a} t}$ (the other terms are nonsynchronous and give no average interaction) results in

$$
\begin{aligned}
e^{j \omega_{a} t} & \frac{d D_{a}^{*}}{d t} \\
= & -\frac{1}{4}\left\{S_{a, a+1}^{0} \sqrt{\frac{\omega_{a+1}}{\omega_{a}}}\left(j \omega_{a+1}-j \omega\right) D_{a+1}^{*} e^{i\left[\left(\omega_{a+1}-\omega\right) t-\phi 1\right.}\right. \\
& \left.+S_{a, a-1}^{0} \sqrt{\frac{\omega_{a-1}}{\omega_{a}}}\left(j \omega_{a-1}+j \omega\right) D_{a-1}^{*} e^{i\left[\left(\omega_{a-1}+\omega\right) t+\phi\right]}\right\}
\end{aligned}
$$

which after using (31) becomes

$$
\begin{aligned}
\frac{d D_{a}^{*}}{d t}=-\frac{1}{4}\left(j S_{a, a+1}^{0}\right. & \sqrt{\omega_{a+1} \omega_{a}} e^{-i \phi} D_{a+1}^{*} \\
& \left.+j S_{a, a-1}^{0} \sqrt{\omega_{a-1} \omega_{a}} e^{+i \phi} D_{a-1}^{*}\right) .
\end{aligned}
$$

Defining a coupling coefficient $\kappa_{a b}$ by

$$
\kappa_{a b}=\left(\omega_{a} \omega_{b}\right)^{1 / 2} \frac{S_{a b}^{0}}{2}=\left(\omega_{a} \omega_{b}\right)^{1 / 2} \int_{V_{0}} \frac{\epsilon_{1}(\bar{r})}{2 \epsilon} \bar{E}_{a} \cdot \bar{E}_{b} d v
$$

and approximating $\sqrt{\omega_{a+1} \omega_{a}}$ in the region of interest by $\omega_{a}$, (33) can be rewritten as

$$
\frac{d D_{a}^{*}}{d t}=-j \frac{\kappa}{2} e^{-j \phi} D_{a+1}^{*}-j \frac{\kappa}{2} e^{+j \phi} D_{a-1}^{*} .
$$

These are the equations of motion for the normal mode amplitudes of an optical resonator modulated "on resonance" ( $\omega=\omega_{a+1}-\omega_{a}$ ). Equation (35) is identified with a familiar Bessel equation recursion formula ${ }^{2}$ so that its solution can be written directly as

$$
D_{a}^{*}(t)=e^{-j a(\phi+\pi / 2)} J_{a}(\kappa t)
$$

where $J_{a}$ is the ordinary Bessel function of order $a$. The solution including the $e^{i \omega_{a} t}$ term is thus

$$
c(t)=\sum_{-\infty}^{+\infty} J_{a}(\kappa t) e^{j a(\phi+\pi / 2)} e^{j \omega_{a} t}
$$

and constitutes a transient FM oscillation mode with a modulation index $\kappa t$ and a center frequency $\omega_{0}$. This mode of oscillation is discussed in Peterson and Yariv [5], Yariv [10], Harris and McDuff [13], and by Harris and McDuff [14] which takes into account nonlinear behavior and mode competition effects.

Another mode of FM oscillation results when the dielectric modulation frequency is nearly, but not quite, equal to the intermode spacing $\omega_{a+1}-\omega_{a}$ of the laser resonator. The deviation from resonance is taken as $\Delta \omega$ so that

$$
\omega_{a+1}-\omega_{a}=\omega-\Delta \omega
$$

2See, for example, P. M. Morse and H. Feshbach, Methods of Mathematical Physics. New York: MeGraw-Hill, 1953, pt. 2, p. 1323. 
Using the substitution

$$
\left.D_{a}^{*}(t)=G_{a}^{*}(t) e^{i(a \Delta \omega t+a \phi}\right)
$$

in (32) yields

$$
\frac{d G_{a}^{*}}{d t}+j a \Delta \omega G_{a}^{*}=-\frac{j \kappa}{2}\left(G_{a+1}^{*}+G_{a-1}^{*}\right)
$$

where we made use of (34) and neglected, again, the small dependence of $\kappa$ on the optical frequency in the region of interest.

The steady-state solution of $(40)$ is

$$
G_{a}^{*}=(-1)^{a} J_{a}\left(\frac{\kappa}{\Delta \omega}\right)
$$

The complete optical field can be written as

$c(t)=\sum_{a=-\infty}^{+\infty} c_{a}^{*}(t)=\sum_{a}(-1)^{a} J_{a}\left(\frac{\kappa}{\Delta \omega}\right) e^{i\left[\left(\omega_{a}+a \Delta \omega\right) t+a \phi\right]}$,

and is to be viewed as the basic resonator mode in the presence of a modulated $\epsilon . c(t)$ corresponding according to (42), to an FM oscillation with a center frequency $\omega_{0}$ and a modulation index $(\kappa / \Delta \omega)$ [10], [13]. Frequency modulation of optical lasers in which the modulation of $\epsilon$ is caused by modulating an electro-optic crystal inside the laser resonator was proposed by Yariv [4], [10]. Revelant experiments were performed by Peterson and Yariv [5] and by Harris and Targ [6]. Harris and McDuff [14] have extended the theory to the nonlinear region and considered the effects of gain saturation and mode competition. Some detailed FM laser experiments have been described by by Amman et al. [18].

\section{Parametric Amplification and Oscillation}

Another situation which may be treated as a special case of the formalism developed above is that of parametric oscillation (or amplification) in a multimode resonator. The starting point is, again, (30). We assume that the interaction is limited to two modes; a "signal" mode at $\omega_{1}$ and an "idler" mode at $\omega_{2}$. The dielectric modulation frequency $\omega$ is equal to the sum of $\omega_{1}$ and $\omega_{2}$

$$
\begin{gathered}
\epsilon_{1}(\bar{r}, t)=\epsilon_{1}(\bar{r}) \cos (\omega t+\phi) \\
\omega=\omega_{1}+\omega_{2} .
\end{gathered}
$$

For the case of two modes (30) becomes

$$
\begin{aligned}
\frac{d D_{1}^{*}}{d t} e^{i \omega_{1} t}= & -\frac{j \omega_{2}}{4} \sqrt{\frac{\omega_{2}}{\omega_{1}}} S_{1,2} e^{j\left(\omega t+\phi-\omega_{2} t\right)} D_{2} \\
& +\frac{j \omega}{4} \sqrt{\frac{\omega_{2}}{\omega_{1}}} S_{1,2} e^{j\left(\omega t+\phi-\omega_{2} t\right)} D_{2}
\end{aligned}
$$

${ }^{3}$ In the optical region this discrimination is due to the fact that the coupling coefficient $S_{1,2}$ (or $\kappa_{1,2}$ ) depends on the direction of propagation in the nonlinear crystals which is modulated dielectrically and will usually be vanishingly small except for one pair of $\omega_{1}, \omega_{2}$. In the microwave region the mode spacing, for small cavities, is nonequal and coupling between more than two modes can be avoided. where the "slow" terms involving $\dot{D}_{1}$ and $\dot{D}_{2}$ on the right side of (30) have been neglected. Using (43) gives

$$
\begin{aligned}
& \frac{d D_{1}^{*}}{d t}=j \frac{\kappa}{2} D_{2} e^{+j \phi} \\
& \frac{d D_{2}}{d t}=-j \frac{\kappa}{2} D_{1}^{*} e^{-i \phi}
\end{aligned}
$$

where $\kappa=\kappa_{1,2}$ as defined by (34).

The solution of $(42)$ is

$$
\begin{aligned}
& D_{1}^{*}(t)=D_{1}^{*}(0) \cosh \frac{\kappa t}{2}+j e^{+j \phi} D_{2}(0) \sinh \frac{\kappa t}{2} \\
& D_{2}(t)=D_{2}(0) \cosh \frac{\kappa t}{2}-j e^{-i \phi} D_{1}^{*}(0) \sinh \frac{\kappa t}{2} .
\end{aligned}
$$

Equations (45) describe the temporal buildup of oscillation in a lossless parametric oscillator.

To obtain an expression for the threshold of parametric oscillation we must take cognizance of the losses that, up to this point, have been neglected. This can be done formally by introducing an effective conductivity $\sigma$ in the analysis with $\sigma / \epsilon$ equal to the decay lifetime in the mode. This introduces an extra term $(-\sigma / 2 \epsilon) c_{a}^{*}$ on the right side of (26) which eventually shows up as

$$
\begin{aligned}
& \frac{d D_{1}^{*}}{d t}=-\frac{\omega_{1}}{2 Q_{1}} D_{1}^{*}+j \frac{\kappa}{2} D_{2} e^{j \phi} \\
& \frac{d D_{2}}{d t}=-\frac{\omega_{2}}{2 Q_{2}} D_{2}-j \frac{\kappa}{2} D_{1}^{*} e^{-i \phi}
\end{aligned}
$$

where the conventional substitution $\sigma / \epsilon=\omega / Q$ has been made. The $Q$ factors can now be considered as representing all the mode losses including that of external coupling.

The start-oscillation condition is derived by putting $d D_{1}^{*} / d t=d D_{2} / d t=0$. The determinantal equation for nontrivial solution for $D_{1}^{*}$ and $D_{2}$ gives

$$
\frac{\omega_{1} \omega_{2}}{Q_{1} Q_{2}}=\kappa^{2}
$$

which using (34) with $a=1, b=2$, becomes

$$
\int_{V_{a}} \frac{\epsilon_{1}(\bar{r})}{2 \epsilon} \bar{E}_{1} \cdot \bar{E}_{2} d v=\frac{1}{\sqrt{Q_{1} Q_{2}}} .
$$

This is the start-oscillation condition. It may be compared with the corresponding lumped-circuit expression for parametric oscillation that reads

$$
\frac{\Delta c}{2 \sqrt{c_{1} c_{2}}}=\frac{1}{\sqrt{Q_{1} Q_{2}}}
$$

where $\Delta c$ is the amplitude of the time-varying part of the circuit capacitance, $c_{1}$ and $c_{2}$ are, respectively, the capacitances in the signal and idler circuits.

In the optical parametric oscillator the modulation of the dielectric constant is brought about by applying an intense "pump" optical field $E_{p 0}(\bar{r}) \cos \omega t$ to a nonlinear crystal medium characterized by the nonlinear suscepti- 
bility tensor elements $\chi_{i j k}[15],[17]$. This modulation can be considered as an effective modulation of the dielectric constant $\epsilon_{1}(\bar{r})$ according to $\epsilon_{1}(\bar{r})=\chi E_{p 0}(\bar{r})$ where $\chi$ is the appropriate $\chi_{i j k}$ element.

This point of view has been justified in a detailed analysis of the optical parametric oscillator [20].

It may be interesting to estimate the threshold requirements in terms of known materials and available pump powers. When the index matching conditions are fulfilled [19], [20], the spatial integral of (37) is equal to unity and the threshold condition becomes

$$
\frac{\delta \epsilon}{2 \epsilon}=\frac{\chi E_{p 0}}{2 \epsilon} \geq \frac{1}{\sqrt{Q_{1} Q_{2}}},
$$

since the nonlinear coefficients $\chi$ are usually quoted in egs units we use the equivalent cgs-threshold expression

$$
\frac{2 \pi \chi E_{p 0}}{\epsilon} \geq \frac{1}{\sqrt{Q_{1} Q_{2}}}
$$

In a typical optical resonator with a length of $5 \mathrm{~cm}$, a loss per pass of one percent, operating at a frequency of $3 \times 10^{14} \mathrm{c} / \mathrm{s}(1 \mu)$, the quality factor $Q_{1} \sim Q_{2}$ is of the order of magnitude of $10^{8}$. Choosing, as an example, $\mathrm{KH}_{2} \mathrm{PO}_{4}(\mathrm{KDP})$ as the nonlinear crystal, the appropriate nonlinear constant is [21] $\chi_{z x y} \sim 3 \times 10^{-9}$. Substituting these values and $\epsilon \approx 2.2$, into (48) gives

$$
E_{p 0} \approx 2 \times 10^{-1} \text { esu }
$$

for the threshold pump field. The corresponding power density is $5 \times 10^{3}$ watts $/ \mathrm{cm}^{2}$, a number easily attained with pulsed lasers. If instead of KDP we calculate the threshold pump field for a crystal such as $\mathrm{LiNbO}_{3}$ with $x \approx 3 \times 10^{-8}$ esu [22], the result is

$$
E_{p 0} \approx 2 \times 10^{-2} e s u
$$

which corresponds to a threshold power density of $\sim 50$ watts $/ \mathrm{cm}^{2}$. The last result indicates that a $\mathrm{CW}$ parametric oscillator is quite feasible. This power density is easily available inside the optical resonator of present day gaseous laser oscillators. This suggests incorporating the nonlinear crystal into the laser oscillator for parametric $\mathrm{CW}$ oscillation. A pulsed parametric oscillator using $\mathrm{LiNbO}_{3}$ has been described by Giordmaine and Miller [9].

\section{Loss Modulation}

Another class of parametric interactions results when a lossy, rather than reactive, parameter is modulated harmonically. A form of loss modulation was employed by Hargrove et al. [3] who introduced an acoustic diffraction cell into the optical resonator of a laser oscillator. The working equations for this case and a discussion of their implication have been given by the author [10]. This section includes the derivation and some additional discussion.

The modulation of loss will be introduced by allowing the effective conductivity $\sigma$ of the resonator medium to vary in space and time so that Maxwell equations can be written as

$$
\begin{aligned}
& \bar{\nabla} \times \bar{H}=\sigma(\bar{r}, t) \bar{E}+\epsilon \frac{\partial \bar{E}}{\partial t} \\
& \bar{\nabla} \times \bar{E}=-\mu \frac{\partial \bar{H}}{\partial t} .
\end{aligned}
$$

Substituting for $\bar{H}$ and $\bar{E}$ their expansion according to (6) and using (3) results in

$\sum_{a} \frac{1}{\sqrt{\mu_{0}}} \omega_{a} q_{a} k_{a} \bar{E}_{a}$

$$
=-\frac{\sigma(\bar{r}, t)}{\sqrt{\epsilon}} \sum_{a} p_{a} \bar{E}_{a}-\sqrt{\epsilon} \sum_{a} \dot{p}_{a} \bar{E}_{a}
$$

for the first equation of (49) and in

$$
\dot{q}_{b}=p_{b}
$$

for the second.

Taking the dot product of (40) with $\bar{E}_{b}$ and integrating over the cavity volume leads to

$$
\omega_{b}^{2} q_{b}=-\sum_{a} S_{a, b}(t) p_{a}-\dot{p}_{b}
$$

where

$$
S_{a, b}(t)=\frac{1}{\epsilon} \int_{V_{s}} \sigma(\bar{r}, t) \vec{E}_{a} \cdot \bar{E}_{b} d t .
$$

Equations (51) and (52) are the equations of motion for the $p_{b}$ 's and $q_{b}$ 's. By substituting for $p_{b}$ and $q_{b}$ from (15), we obtain, after some rearrangement, the following equations for the normal mode amplitudes:

$$
\begin{aligned}
& \frac{d c_{a}^{*}}{d t}=j \omega_{a} c_{a}^{*}+\sum_{b} \kappa_{b, a}(t)\left(c_{b}^{*}-c_{b}\right) \\
& \frac{d c_{a}}{d t}=-j \omega_{a} c_{a}-\sum_{b} \kappa_{b, a}(t)\left(c_{b}^{*}-c_{b}\right)
\end{aligned}
$$

where

$$
\kappa_{b, a}(t)=\frac{1}{2} S_{b, a}(t) \sqrt{\frac{\omega_{b}}{\omega_{a}}} .
$$

Taking the conductivity as the sum of an average term and an harmonic perturbation

$$
\sigma(\bar{r}, t)=\sigma_{0}+\sigma_{1}(\bar{r}) \cos (\omega t+\phi),
$$

the expression for $\kappa_{b, a}(t)$ becomes

$$
\begin{gathered}
\kappa_{\grave{b}, a}(t)=\frac{\sigma_{0}}{2 \epsilon} \delta_{a b}+\frac{\kappa_{b, a}}{2 \epsilon}\left[e^{j(\omega t+\phi\rangle}+e^{-j(\omega t+\phi)}\right] \\
\kappa_{b, a}=\frac{1}{2 \epsilon} \sqrt{\frac{\omega_{b}}{\omega_{a}}} \int_{V_{o}} \sigma_{1}(\bar{r}) \bar{E}_{a} \cdot \bar{E}_{b} d v .
\end{gathered}
$$

A substitution of (55) into the equation of motion (54) gives

$$
\begin{aligned}
\frac{d c_{a}^{*}}{d t}=j \omega_{a} c_{a} & -\frac{\sigma_{0}}{2 \epsilon}\left(c_{a}^{*}-c_{a}\right) \\
& +\sum_{b} \frac{\kappa_{b, a}}{2}\left[e^{j(\omega t+\phi)}+e^{-j(\omega t+\phi)}\right]\left(c_{b}^{*}-c_{b}\right)
\end{aligned}
$$


and its complex conjugate for $d c_{a} / d t$. These are the main working equations.

Consider first the case when the modulation frequency $\omega$ is equal to the mode spacing (or a multiple thereof) of the optical resonator. It is further assumed that the resonance frequencies are equally spaced so that

$$
\omega_{a+1}-\omega_{a}=\omega_{a}-\omega_{a-1}=\omega .
$$

Substituting (58) into (57) and retaining on the right side only the synchronous terms, i.e., those having $\exp \left(j \omega_{a} t\right)$ time dependence, gives

$$
\begin{aligned}
\frac{d c_{a}^{*}}{d t}=\left(j \omega_{a}-\frac{\sigma_{0}}{2 \epsilon}\right) c_{a}^{*} & +\frac{\kappa_{a, a+1}}{2} e^{-j(\omega t+\phi)} c_{a+1}^{*} \\
& +\frac{\kappa_{a, a-1}}{2} e^{j(\omega t+\phi)} c_{a-1}^{*} .
\end{aligned}
$$

A substitution of

$$
c_{a}^{*}(t)=D_{a}^{*}(t) e^{j\left[\left(\omega_{a}+j \sigma_{0} / 2 \epsilon\right) \delta+\alpha \phi\right]}
$$

and taking $\kappa_{a, a+1}=\kappa_{a, a-1}=\kappa$

$$
\frac{d D_{a}^{*}}{d t}=\frac{\kappa}{2}\left(D_{a+1}^{*}+D_{a-1}^{*}\right)
$$

whose solution is [20]

$$
D_{a}^{*}(t)=I_{a}(\kappa t)
$$

which upon substitution in (60) yields

$$
c_{a}^{*}(t)=I_{a}(\kappa t) e^{j\left[\left(\omega_{0}+a \omega\right) t+a \phi\right]} e^{-\left(\sigma_{0} / 2 \epsilon\right) t}
$$

where $\omega_{0}$ is the frequency of the reference mode, $a=0$.

Another situation of practical importance, discussed in Yariv [10], results when the modulation frequency is slightly off resonance, i.e., when

$$
\omega_{x+1}-\omega_{a}=\omega-\Delta \omega
$$

where $\Delta \omega$ is the deviation from resonance. Defining the variable $D_{a}^{*}(t)$ by

$$
c_{a}^{*}=D_{a}^{*}(t) e^{i[(\omega a+\alpha \Delta \omega) t+a \phi+a \pi / 2]} e^{-\left(\sigma_{0} / 2 \epsilon\right) t}
$$

and substituting into (59), using

$$
\omega_{a+1}-\omega_{a}=\omega-\Delta \omega,
$$

gives

$$
\frac{d D_{a}^{*}}{d l}+j a \Delta \omega D_{a}^{*}=j \frac{\kappa}{2} D_{a+1}^{*}-j \frac{\kappa}{2} D_{a-1}^{*} .
$$

whose steady-state solution $\left(d D_{a}^{*} / d t=0\right)$ is

$$
D_{a}^{*}=I_{a}\left(\frac{\kappa}{\Delta \omega}\right)
$$

so that $c_{a}^{*}(t)$ is given by [10]

$$
c_{a}^{*}(t)=I_{a}\left(\frac{\kappa}{\Delta \omega}\right) e^{i\left[\left(\omega_{c}+a \Delta \omega\right) t+a \phi+a x / 21\right.} .
$$

The new "super mode"

$$
c(t)=\sum_{a} c_{a}(t)
$$

where $c_{a}(t)$ is given by (64), corresponds to an optical field at $\omega_{0}$ whose envelope is made up of a train of pulses with a period of $2 \pi / \omega[10]$, [23].

\section{Parametric Oscillation by Loss Modulation}

Since reactive parametric modulation can, as discussed in Section IV, give rise to oscillation, it is of interest to explore, at least on paper, the possibility of oscillation via loss modulation.

The starting point is (57) with the modulation frequency $\omega$ put equal to the sum of two resonance frequencies that are taken as $\omega_{1}$ and $\omega_{2}$

$$
\omega=\omega_{1}+\omega_{2} \text {. }
$$

After substituting (65) in (57) and defining $D_{a}^{*}(t)$ by

$$
c_{a}^{*}(t)=D_{a}^{*}(t) e^{j \omega_{a} t}
$$

the synchronous part of (57) becomes

$$
\begin{aligned}
& \frac{d D_{1}^{*}}{d t}=-\frac{\sigma_{01}}{2 \epsilon} D_{1}^{*}-\frac{\kappa_{2,1}}{2} e^{j \phi} D_{2} \\
& \frac{d D_{2}}{d t}=-\frac{\sigma_{02}}{2 \epsilon} D_{2}-\frac{\kappa_{1,2}}{2} e^{-j \phi} D_{1}^{*}
\end{aligned}
$$

so that $D_{1}^{*}$ couples to $D_{2}$ and vice versa. The steady-state oscillation condition results in a determinantal equation

$$
\kappa_{2,1} \kappa_{1.2}=\frac{\sigma_{01} \sigma_{02}}{\epsilon^{2}} .
$$

Under ordinary conditions when $\sigma_{01}$ and $\sigma_{02}$ represent the passive losses of modes " 1 ". and " 2 ", respectively, (68) cannot be fulfilled. This follows from (56), which shows that $\kappa_{b, a} \leq(1 / 2 \epsilon) \sqrt{\omega_{b} / \omega_{a}} \sigma_{1}$ and from the fact that the modulated part of the conductivity $\sigma_{1}$ must satisfy the condition $\sigma_{1}<\sigma_{0}$, where $\sigma_{0}$ is the average conductivity. Equation (68) can be satisfied, however, when the average losses of mode " 1 " (represented by $\sigma_{01}$ ) or of mode " 2 " $\left(\sigma_{02}\right)$ are reduced without a similar reduction in the modulated conductivity $\sigma_{1}$. This could be the case if the total conductivity of mode 1 , for example, is given by

$$
\sigma=\sigma_{01}^{\prime}+\sigma_{1}(\bar{r}) \cos (\omega t+\phi)-\sigma_{01}^{\prime \prime}
$$

where $\sigma_{01}^{\prime}$ represents the passive resonator losses at $\omega_{1}$ and satisfies the condition $\sigma_{01}^{\prime}>\sigma_{1}(\bar{\gamma})$. The term $-\sigma_{01}^{\prime \prime}$ represents some gain mechanism at $\omega_{1}$, such as that due to an inverted laser population. Under these conditions $\sigma_{01}$ in $(68)$ is given by

$$
\sigma_{01}=\sigma_{01}^{\prime}-\sigma_{01}^{\prime \prime}
$$

and can, in principle, be made small enough so that the condition of (68) for parametric oscillation obtains.

In the case of reactive modulation it, is well known that the numbers of photons generated at the various frequencies satisfy the Manley-Row relations [24]. In the case of the parametric oscillator this relation takes the form $P_{1} / \omega_{1}=P_{2} / \omega_{2}$ where $P_{1}$ and $P_{2}$ are the total powers 
produced at the signal $\left(\omega_{1}\right)$ and idler $\left(\omega_{2}\right)$ frequencies, respectively. These relations are satisfied by (46).

In the case of loss modulation we get directly from (67)

$$
\begin{aligned}
\frac{d}{d t}\left[\omega_{1} D_{1}^{*}(t) D_{1}(t)\right]= & -\frac{\sigma_{01}}{\epsilon}\left(\omega_{1} D_{1}^{*} D_{1}\right) \\
& -\frac{\kappa_{2,1}}{2} \omega_{1}\left(e^{-j \phi} D_{1}^{*} D_{2}^{*}+e^{j \phi} D_{1} D_{2}\right) \\
\frac{d}{d t}\left[\omega_{2} D_{2}^{*}(t) D_{2}(t)\right] & =-\frac{\sigma_{02}}{\epsilon}\left(\omega_{2} D_{2}^{*} D_{2}\right) \\
& -\frac{\kappa_{1,2}}{2} \omega_{2}\left(e^{-i \phi} D_{1}^{*} D_{2}^{*}+e^{j \phi} D_{1} D_{2}\right) .
\end{aligned}
$$

According to (16) and (66) $\omega_{a} D_{a}^{*} D_{a}$ is the energy stored in the ath mode. The last term in each of the equations in (69) represents the power produced by the loss modulation. Since, according to $(56), \kappa_{2,1} \omega_{1}=\kappa_{1,2} \omega_{2}$, these powers are equal and we can write

$$
P_{1}=P_{2}
$$

where $P_{1}$ and $P_{2}$ are the powers generated by the nonlinear loss modulation at $\omega_{1}$ and $\omega_{2}$, respectively.

As pointed out in Louisell et al. [2], the parametric equations in the time domain have the same form as the corresponding spatial equations. As an example consider (67). The spatial equivalent of these equations is

$$
\begin{aligned}
& \frac{d D_{1}^{*}}{d z}=-\frac{1}{2} \alpha_{1} D_{1}^{*}-\frac{c_{2} \cdot 1}{2} e^{j \phi} D_{2} \\
& \frac{d D_{2}}{d z}=-\frac{1}{2} \alpha_{2} D_{2}-\frac{c_{1.2}}{2} e^{-j \phi} D_{1}^{*},
\end{aligned}
$$

where $\alpha_{j}=\sigma_{0 j} / \epsilon c, c$ being the velocity of propagation, $\alpha$ is the spatial attenuation constant, and $c_{i, j}$ is related to $\kappa_{i, j}$ by

$$
c_{i, i}=\kappa_{i, j} / c
$$

when index matching [9] obtains. From (70) it follows that

$$
\frac{d^{2} D_{1}^{*}}{d z^{2}}=\left(\frac{\alpha_{1}^{2}}{4}+\frac{c_{2,1} c_{1,2}}{4}\right) D_{1}^{*}+\left(\alpha_{1}+\alpha_{2}\right) c_{2,1} e^{j \phi} D_{2} .
$$

Consider the special case when $\alpha_{1}+\alpha_{2}=0$, i.e., when the negative losses (gain) of one wave are equal in magnitude to the losses of the other. When this happens (71) has a simple solution given by

$$
\begin{aligned}
D_{1}^{*}(z)= & D_{1}^{*}(0) \cosh (\gamma z) \\
& -\left[\frac{\alpha_{1}}{2 \gamma} D_{1}^{*}(0)+\frac{c_{2,1}}{2 \gamma} e^{i \phi} D_{2}(0)\right] \sinh (\gamma z) \\
D_{2}(z)= & D_{2}(0) \cosh (\gamma z) \\
& -\left[\frac{\alpha_{2}}{2 \gamma} D_{2}(0)+\frac{c_{1,2}}{2 \gamma} e^{-i \phi} D_{1}^{*}(0)\right] \sinh (\gamma z)
\end{aligned}
$$

where

$$
\gamma=\frac{1}{2}\left(\alpha_{1}^{2}+c_{1 ; 2} c_{2,1}\right)^{1 / 2} .
$$

Taking the case of a single input at $\omega_{1}$ so that $D_{1}^{*}(0) \neq 0$ but $D_{2}(0)=0,(72)$ becomes

$$
\begin{aligned}
& D_{1}^{*}(z)=D_{1}^{*}(0) \cosh (\gamma z)-\frac{\alpha_{1}}{2 \gamma} D_{1}^{*}(0) \sinh (\gamma z) \\
& D_{2}(z)=-\frac{c_{1,2}}{2 \gamma} e^{-i \phi} D_{1}^{*}(0) \sinh (\gamma z) .
\end{aligned}
$$

Equations (73) describe how the amplitude of the input wave at $\omega_{1}$ is amplified by a factor $\sim\left(1-\alpha_{1} / 2 \gamma\right) e^{\gamma z}$ for $\gamma z \gg 1$. In addition, a new wave at $\omega_{2}=\omega-\omega_{1}$ is generated so that the output can be taken at $\omega_{1}$ or $\omega_{2}$.

Equations (73) satisfy the condition $P_{1}=P_{2}$ derived in the preceding section. In this case it takes the form

$$
\left[D_{1}(z) D_{1}^{*}(z)-D_{1}(0) D_{1}^{*}(0)\right] \omega_{1}=D_{2}(z) D_{2}^{*}(z) \omega_{2} .
$$

\section{Parametric Frequency Conversion by Dielectric Modulation}

In the parametric frequency converter the modulation frequency $\omega$ fulfills the condition

$$
\omega_{2}=\omega+\omega_{1}
$$

so that it is equal to the difference of the two frequencies that it couples and not to their sum. When (74) applies, the synchronous part of $(30)$ becomes

$$
\begin{aligned}
& \frac{d D_{2}^{*}}{d t}=-j \frac{\kappa}{2} e^{j \phi} D_{1}^{*} \\
& \frac{d D_{1}^{*}}{d t}=-j \frac{\kappa}{2} e^{-j \phi} D_{2}^{*}
\end{aligned}
$$

where $\kappa$ is defined, as in (34), by

$$
\kappa=\kappa_{1,2}=\kappa_{2,1}=\left(\omega_{1} \omega_{2}\right)^{1 / 2} \int_{V_{c}} \frac{\epsilon_{1}(\bar{r})}{2 \epsilon} \bar{E}_{a} \cdot \bar{E}_{b} d v .
$$

Equations (75) do not include losses. Their solution, as is well known, [2], [16], and [17], corresponds to a periodic exchange of energy between modes 1 and 2 described by

$$
\begin{aligned}
& D_{1}^{*}(t)=D_{1}^{*}(0) \cos \left(\frac{\kappa t}{2}\right)-j e^{-j \phi} D_{2}^{*}(0) \sin \left(\frac{\kappa t}{2}\right) \\
& D_{2}^{*}(t)=D_{2}^{*}(0) \cos \left(\frac{\kappa t}{2}\right)-j e^{j \phi} D_{1}^{*}(0) \sin \left(\frac{\kappa t}{2}\right)
\end{aligned}
$$

where, according to (16), the energy in a mode, say mode $a$, is given by $\omega_{a} c_{a} c_{a}^{*}=\omega_{a} D_{a} D_{a}^{*}$. It is of interest to investigate the effect of the inclusion of losses (positive and negative) on the behavior of the frequency converter. This is done by adding, phenomenologically, a dissipative term that accounts for the decay (or growth) of radiation density in each mode at the absence of coupling. Equation (75) is rewritten as

$$
\begin{aligned}
& \frac{d D_{1}^{*}}{d t}=-\frac{\sigma_{01}}{2 \epsilon} D_{1}^{*}-j \frac{\kappa}{2} e^{-i \phi} D_{2}^{*} \\
& \frac{d D_{2}^{*}}{d t}=-\frac{\sigma_{02}}{2 \epsilon} D_{2}^{*}-j \frac{\kappa}{2} e^{j \phi} D_{1}^{*}
\end{aligned}
$$


where $\sigma_{01}$ and $\sigma_{02}$ are the effective conductivities at $\omega_{1}$ and $\omega_{2}$. The determinantal equation resulting from the steady-state condition is

$$
\frac{\sigma_{01} \sigma_{02}}{\epsilon^{2}}=-\kappa^{2}
$$

A steady-state oscillation is thus only possible when one of the two modes has (sufficient) negative losses. This can be accomplished, for example, by having one of the modes amplified by a laser transition simultaneously with the dielectric modulation.

\section{Parametric Frequency Conversion by Loss Modulation}

Assume that the frequency $\omega$ at which the losses are modulated is equal to the difference of the frequencies of the two resonances that are coupled by it, i.e.,

$$
\omega_{2}=\omega+\omega_{1}
$$

The synchronous part of (57) becomes

$$
\begin{aligned}
& \frac{d c_{1}^{*}}{d t}=j \omega_{1} c_{1}^{*}-\frac{\sigma_{01}}{2 \epsilon} c_{1}^{*}+\frac{\kappa_{2,1}}{2} e^{-j(\omega t+\phi)} c_{2}^{*} \\
& \frac{d c_{2}^{*}}{d t}=j \omega_{2} c_{2}^{*}-\frac{\sigma_{02}}{2 \epsilon} c_{2}^{*}+\frac{\kappa_{1,2}}{2} e^{j(\omega t+\phi)} c_{1}^{*}
\end{aligned}
$$

which after substituting $c_{a}^{*}(t)=D_{a}^{*}(t) e^{j \omega_{\star} t}$ becomes

$$
\begin{aligned}
& \frac{d D_{1}^{*}}{d t}=-\frac{\sigma_{01}}{2 \epsilon} D_{1}^{*}+\frac{\kappa_{2,1}}{2} e^{-j \phi} D_{2}^{*} \\
& \frac{d D_{2}^{*}}{d t}=-\frac{\sigma_{02}}{2 \epsilon} D_{2}^{*}+\frac{\kappa_{1,2}}{2} e^{j \phi} D_{1}^{*} .
\end{aligned}
$$

The determinantal equation resulting from the steadystate oscillation condition is

$$
\frac{\sigma_{01} \sigma_{02}}{\epsilon}=\kappa_{2,1} \kappa_{1,2}
$$

and is the same as that for the loss modulated parametric oscillator, (68). The argument following (68) applies, consequently, in this case and shows that in the presence of sufficient negative losses simultaneous oscillation at $\omega_{1}$ and $\omega_{2}$ can be sustained by "lossy" pumping at $\omega=$ $\omega_{2}-\omega_{1}$.

In a manner similar to that discussed in Section VI it should be possible to make a spatially distributed frequency converter for converting a "low"-frequency input at $\omega_{1}$ to an output wave at $\omega_{2}=\omega+\omega_{1}$. This may be especially useful for converting a low-frequency (say infrared) signal to a visible (or near visible) one where it can be detected efficiently and with a fast response time with conventional photoemissive detectors.

\section{Conchusion}

The equations of motion governing the interaction of optical modes in the presence of time-varying parameters have been derived. A formalism of normal modes is developed which results in concise and symmetric formulation of the problem. Two general types of modulation have been considered: 1) modulation of the dielectric constant and 2) modulation of the losses. In addition to treating some well-known cases such as parametric oscillation and internal mode-locking in laser oscillators, new interactions involving loss modulation and dielectric modulation in the presence of negative losses have been considered.

\section{REFERENCES}

[1] W. H. Louisell, Coupled Modes and Parametric Electronics. New York: Wiley, 1960.

[2] W. H. Louisell, A. Yariv, and A. E. Siegman, "Quantum fluctuations and noise in parametric processes," Phys. Rev., vol. 124 , pp. 1646-1654, December 1961. This reference is denoted in the text as LYS.

[3] L. E. Hargrove, R. L. Fork, and M. A. Pollack, "Locking of $\mathrm{He}-\mathrm{Ne}$ laser modes induced by synchronous intracavity modulation," 1964, Annual Conference on Electron Device Research, Cornell University, Ithaca, N. Y.; Appl. Phys. Letters, vol. 5, p. 45, July 1964 .

[4] A. Yariv, "Electro-optic frequency modulation in optical resonators," Proc. IEEE (Correspondence), vol. 52, pp. 719-720, June 1964 .

[5] D. G. Peterson and A. Yariv, "Parametric frequency conversion by the electro-optic effect in KDP," Appl. Phys. Letters, vol. 5, pp. 184-186, November 1964.

[6] S. E. Harris and R. Targ, "FM oscillation of the He-Ne laser," A ppl. Phys. Letters, vol. 5, pp. 202-204, November 1964.

[7] R. H. Kingston, "Parametric amplification and oscillation at optical frequencies," Proc. IRE (Correspondence), vol. 50, p.472, April 1962.

[8] N. M. Kroll, "Parametric amplification in spatially extended media and application to the design of tunable oscillators at optical frequencies," Phys. Rev., vol. 127, pp. 1207-1211, August 1962.

[9] J. A. Giordmaine and R. C. Miller, "Tunable coherent parametric oscillation in $\mathrm{LiNbO}_{3}$ at optical frequencies," Phys. Rev. Letters, vol. 14, pp. 973-976, June 1965 .

[10] A. Yariv, "Internal modulation in multimode laser oscillators," 1964 Annual Conf. on Electron Device Research, Cornell University, Ithaca, N. Y., June 1964. Published in J. Appl. Phys., vol. 36, pp. 388-391, February 1965. This and [4] constitute, to the author's knowledge, the first proposal and analysis of an FM laser using electro-optic modulation and mode locking inside a laser resonator.

[11] J. C. Slater, Microwave Electronics. Princeton, N. J.: Van Nostrand, p. 57.

[12] E. Jahnke and F. Emde, Tables of Functions. New York: Dover,

[13] S. E. Harris and O. P. McDuff, "FM laser oscillation-theory," A ppl. Phys. Letters, vol. 5, pp. 205-206, November 1964.

[14] S. E. Harris and O. P. MCDuff, "Theory of FM laser oscillation," IEEE J. of Quantum Electronics, vol. 1, pp. 245-263, September 1965.

[15] P. A. Franken and J. F. Ward, "Optical harmonics and nonlinear phenomena," Rev. Mod. Phys., vol. 35, pp. 23-39, Jan-

[16] $\mathrm{P}$. K. Tien, "Parametric amplification and frequency mixing in propagating circuits," J. Appl. Phys., vol. 29, pp. 1347-1357, September 1958 .

[17] J. A. Armstrong, N. Bloembergen, J. Ducuing, and P. S. Pershan, "Interactions between light waves in nonlinear media," Phys. Rev., vol. 127, pp. 1918-1938, September 1962.

[18] E. O. Amman, B. J. McMurtry, and M. K. Oshman, "Detailed experiments on He-Ne FM lasers," IEEE J. of Quantum Electronics, vol. 1, pp. 263-273, September 1965 .

[19] J. A. Giordmaine, "Mixing of light beams in crystals," Phys. Rev., vol. 8, pp. 19-20, January 1962.

[20] A. Yariv and W. H. Louisell, "Theory of the optical parametric oscillator," to be published.

[21] A. Ashkin, G. D. Boyd, and J. M. Dziedzic, "Observation of continuous optical harmonic generation with gas masers," Phys. Rev. Letters, vol. 11, p. 14, July 1963.

[22] G. D. Boyd, R. C. Miller, K. Nassau, W. L. Bond, and A. Savage, "LiNbO ${ }_{3}$ an efficient phase matchable nonlinear optical material," Appl. Phys. Letters, vol. 5, pp. 234-236, December 1964.

[23] M. DiDomenico, Jr., "Small-signal analysis of internal (coupling-type) modulation of lasers," $J$. Appl. Phys., vol. 35, pp. 2870-2876, October 1964.

[24] J. M. Manley and H. E. Rowe, "General energy relations in nonlinear reactance," Proc. IRE (Correspondence), vol. 47, pp. 2115-2116, December 1959. 